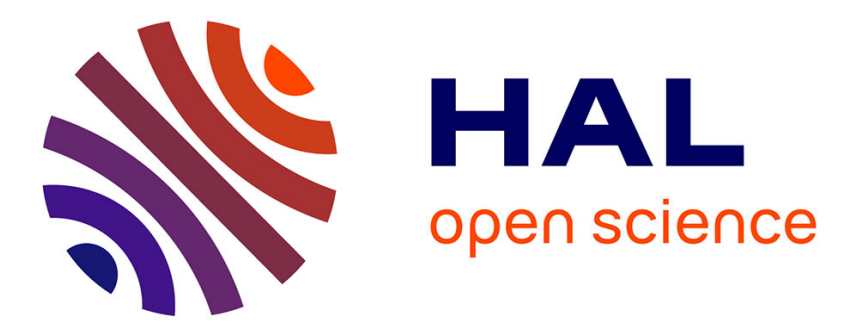

\title{
Modeling Human Postural Coordination to Improve the Control of Balance in Humanoids
}

\author{
Vincent Bonnet, Julien Lagarde, Philippe Fraisse, Nacim Ramdani, Sofiane \\ Ramdani, Philippe Poignet, Benoit Bardy
}

\section{- To cite this version:}

Vincent Bonnet, Julien Lagarde, Philippe Fraisse, Nacim Ramdani, Sofiane Ramdani, et al.. Modeling Human Postural Coordination to Improve the Control of Balance in Humanoids. 7th IEEE-RAS International Conference on Humanoid Robots, Nov 2007, Pittsburgh, PA, United States. pp.324329, 10.1109/ICHR.2007.4813888 . lirmm-00202778

\section{HAL Id: lirmm-00202778 https://hal-lirmm.ccsd.cnrs.fr/lirmm-00202778}

Submitted on 4 Jun 2021

HAL is a multi-disciplinary open access archive for the deposit and dissemination of scientific research documents, whether they are published or not. The documents may come from teaching and research institutions in France or abroad, or from public or private research centers.
L'archive ouverte pluridisciplinaire HAL, est destinée au dépôt et à la diffusion de documents scientifiques de niveau recherche, publiés ou non, émanant des établissements d'enseignement et de recherche français ou étrangers, des laboratoires publics ou privés. 


\title{
Modeling human postural coordination to improve the control of balance in humanoids
}

\author{
V. Bonnet ${ }^{1}$, J. Lagarde ${ }^{2}$, P. Fraisse ${ }^{1}$, N. Ramdani ${ }^{1}$, S. Ramdani ${ }^{2}$, P. Poignet ${ }^{1}$, B. Bardy ${ }^{2,3}$ \\ ${ }^{1}$ LIRMM UMR 5506 CNRS, Univ. Montpellier II, 161 rue Ada, Montpellier, 34392 France \\ ${ }^{2}$ EDM EA 2991, Univ. Montpellier 1, EDM, 700 av. du pic Saint Loup, Montpellier, 34090 France \\ ${ }^{3}$ Institut Universitaire de France, 103 Bd St Michel, Paris, 75005 France
}

\begin{abstract}
This paper models recent data in the field of postural coordination showing the existence of self-organized postural states, and transition between them, underlying supra-postural tracking movements. The proposed biomechanical model, capitalizing on stability and optimization criteria, captures the complex postural behaviors observed in humans and can be used to implement efficient balance control principles in humanoids. Experimental results on humanoids show the relevance of this work.
\end{abstract}

\section{INTRODUCTION}

The field of movement science, perception-action and motor control in humans provides a natural source of inspiration for researchers in humanoid robotics. Due to their anthropomorphic structure, humanoid robots often present dynamic similarities with humans that are interesting to study in both fields of research. The goal of the present paper is to model basic phenomena characterizing human postural coordination, which have motivated in depth analyses during the last decade in the field of human movement science. In 1985, Nasher and Mc Collum [1] observed two postural strategies when the whole body oscillates in upright position. These postural strategies are characterized by a large activity of the ankles during the the ankle strategy, and by the coordinative movements of the hips and the ankles in the hip strategy. Within the framework of coordination dynamics [2], Bardy et al. [3] proposed the use of a collective variable to describe in a simple way the complex biological couplings underlying the ordering in space and time of postural coordination. To identify this collective variables, Bardy et al. [4] used a parametric visual tracking task (Fig. 1).

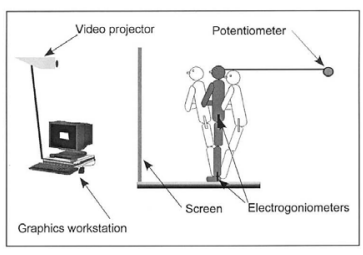

Fig. 1. Task and set-up used in Bardy et al. experiments. Participants faced a white target on a large screen and tracked its motion in the sagittal plane. The frequency of the target increased or decreased continuously.
They had standing participants moving back and forth in the sagittal plane in order to track the displacement of a the target, with the knees locked and the soles in permanent contact with the ground. This simple task allowed the observation of several self-organized properties of the postural system, such as phase transition, multistability, self-organization, critical fluctuations, hysteresis, and critical slowing down. The collective variable able to capture both fully and in a very compact way these properties was the phase difference between the ankle and the hip.

These experimental observations suggest that posture may be organized in terms of dynamical principles [2], and call for the existence of common principles governing pattern formation in complex biological systems. An attempt to reproduce these complex couplings should ameliorate the control of balance in humanoids.

One first approach reaching for this global objective is to ensure the validity of the observed principles in humanoid robots.

A biomechanical model using a double inverted pendulum associated with an under-constraints optimization method was proposed by [5] to reproduce the dynamical and mechanical aforementioned observations. Furthermore the objective function used in [5] was also bio-inspired. [6] has shown that during stance the human body aims at minimizing the variations in energy during tracking tasks. However, this model cannot describe critical slowing down and hysteresis, two important phenomena observed in human behaviors and characterizing non linear systems. The present work has two distinct objectives:

1) develop a biomechanical model reproducing the human observations,

2) implement the model in humanoid robots.

The implementation of the model permits the execution of pre-programmed motion even if balance cannot be maintained, contrary to humans. Humanoid robot sensors provide information to decompose and understand a given task perfectly.

For this purpose, the target tracking task used by [3] and [4] was chosen, and the Martin et al.s optimization model [5] for this task was examined in the context of humanoid robotics.

An analysis of the natural frequencies was performed and compared with the simulations resulting from the combination 
of an optimization and a biomechanical model.

Finally, in order to validate the resulting model, the optimal joint amplitudes were executed in open loop on two different humanoid robots.

\section{BIOMECHANICAL MODEL FOR THE TRACKING TASK}

\section{A. Biomechanical modeling}

Here we replicate the simple biomechanical model proposed by Martin et al. [5] for postural sway in the sagittal plane. The model (Fig. 2) is formed by three rigid segments including the feet, the lower limbs and the upper part of the body which are linked by the ankle and the hip joints (such joints are modeled as frictionless hinges). The entire sole of the feet were assumed to stay in contact with the floor.

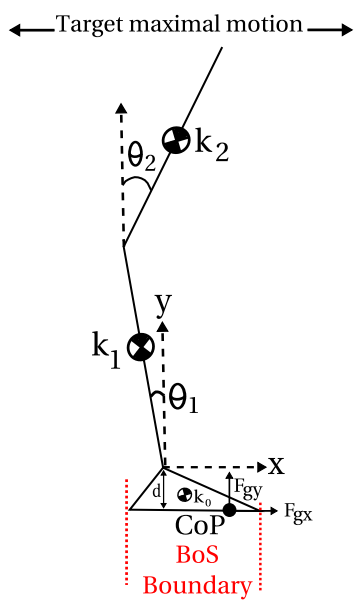

Fig. 2. Biomechanical model in the sagittal plane used to describe human postural coordination

For the inverse dynamic model calculation, the Lagrange movement equations were used. We changed only the joint markers, with respect to the original Martin's model.

$$
\begin{aligned}
\Gamma_{1} & =\ddot{\theta}_{1}\left(m_{1} k_{1}^{2} l_{1}^{2}+l_{1}^{2} m_{2}+I_{1}+I_{2}\right) \\
& +\ddot{\theta}_{2}\left(m_{2} l_{1} l_{2} k_{2} \cos \left(\theta_{1}-\theta_{2}\right)+I_{2}\right) \\
& +\dot{\theta}_{1}^{2}\left(m_{2} l_{1} l_{2} k_{2} \sin \left(\theta_{1}-\theta_{2}\right)\right)+g l_{1} \sin \left(\theta_{1}\right)\left(m_{1} k_{1}+m_{2}\right) \\
\Gamma_{2} & =\ddot{\theta}_{2}\left(m_{2} k_{2}^{2} l_{2}^{2}+I_{2}\right) \\
& +\ddot{\theta}_{1}\left(m_{2} l_{1} l_{2} k_{2} \cos \left(\theta_{1}-\theta_{2}\right)+I_{2}\right) \\
& +\dot{\theta}_{1}^{2}\left(m_{2} l_{1} l_{2} k_{2} \sin \left(\theta_{1}-\theta_{2}\right)\right)+g m_{2} k_{2} l_{2} \sin \left(\theta_{2}\right)
\end{aligned}
$$

where, $\Gamma_{1}, \Gamma_{2}$ represent the torque at the ankle and hip respectively. $\theta_{1}, \dot{\theta_{1}}, \ddot{\theta}_{1}$ are position, velocity and acceleration of joint 1 . Finally, the dynamic parameters of each segment are mass $m$, lenght $l$, center of mass position $k$, inertia $I$, and the gravity constant $g$.

Balance is described by the position of center of pressure $(\mathrm{CoP})$ within the BoS the sagittal plane. It can be expressed as a function of the dynamic parameters (eq.3).

$$
C o P=\frac{\left(\Gamma_{1}-F_{g x} d+m_{0} k_{0} g\right)}{F_{g y}}
$$

where $F_{g x}$ is the horizontal ground reaction force and $F_{g y}$ the vertical ground reaction force. For the calculation of the ground reaction forces, Cahouet et al. [7] proposed to use Euler's equations.

\section{B. Natural frequencies of the double inverted pendulum}

Intrinsic properties of the dynamic model can explain some phenomena observed in experiments, around specific frequencies. Indeed, each mechanical system has natural frequencies at which minimal inputs can provide maximal reactions. This interesting fact has been studied in other research areas such as in humanoid structure construction [9]. Natural frequencies are calculated via the linearization of the inverse dynamic model, taking only the homogeneous equations of the system (4).

$$
\begin{aligned}
& \ddot{\theta}_{1} \alpha_{1}+\ddot{\theta}_{2} \beta_{1}+\theta_{1} \gamma_{1}=0 \\
& \ddot{\theta_{1}} \alpha_{2}+\ddot{\theta}_{2} \beta_{2}+\theta_{1} \gamma_{2}=0
\end{aligned}
$$

and $\alpha, \beta, \gamma$ are coefficients that represent the dynamic parameters; they are defined by the following equations:

$$
\begin{aligned}
\alpha_{1} & =\left(m_{1} k_{1}^{2} l_{1}^{2}+l_{1}^{2} m_{2}+I_{1}+I_{2}\right) \\
\beta_{1} & =\left(m_{2} l_{1} l_{2} k_{2}+I_{2}\right) \\
\gamma_{1} & =g l_{1}\left(m_{1} k_{1}+m_{2}\right) \\
\alpha_{2} & =\left(m_{2} l_{1} l_{2} k_{2}+I_{2}\right) \\
\beta_{2} & =\left(m_{2} k_{2}^{2} l_{2}^{2}+I_{2}\right) \\
\gamma_{2} & =g m_{2} k_{2} l_{2}
\end{aligned}
$$

By considering the periodic solution of (eq. 4), one can solve the system for amplitudes. The associated characteristic equation has two real positive solutions which are the natural frequencies of the system.

$$
\begin{gathered}
\omega_{01}=\frac{-1}{2\left(\alpha_{1} \beta_{2}-\beta_{1} \alpha_{2}\right)}\left(-2\left(\alpha_{1} \beta_{2}-\beta_{1} \alpha_{2}\right)\left(-\gamma_{1} \beta_{2}-\alpha_{1} \gamma_{2}\right.\right. \\
\left.+\sqrt{\left.\left(\gamma_{1} \beta_{2}-\alpha_{1} \gamma_{2}\right)^{2}+4 \gamma_{1} \gamma_{2} \beta_{1} \alpha_{2}\right)}\right)^{1 / 2} \\
\omega_{02}=\frac{-1}{2\left(\alpha_{1} \beta_{2}-\beta_{1} \alpha_{2}\right)}\left(\sqrt { 2 } ( \alpha _ { 1 } \beta _ { 2 } - \beta _ { 1 } \alpha _ { 2 } ) \left(\gamma_{1} \beta_{2}+\alpha_{1} \gamma_{2}\right.\right. \\
\left.\quad+\sqrt{\left.\left(\gamma_{1} \beta_{2}-\alpha_{1} \gamma_{2}\right)^{2}+4 \gamma_{1} \gamma_{2} \beta_{1} \alpha_{2}\right)}\right)^{1 / 2}
\end{gathered}
$$

The numerical values of the natural frequencies for the two tested robots are:

- HRP2: $\omega_{01}=0.48 \mathrm{~Hz}$ and $\omega_{02}=1.05 \mathrm{~Hz}$

- HOAP3: $\omega_{01}=0.74 \mathrm{~Hz}$ and $\omega_{02}=1.65 \mathrm{~Hz}$

The system response shows two resonance peaks at the natural frequencies. This observation tends to confirm the privilege for certain frequencies to naturally decrease the joint power necessary to accomplish the movement, and to increase 
the stability of the system since CoP position is linked to the ankle torque (eq. 3).

\section{Optimal amplitude calculation for the tracking task}

In Bardy et al.'s experiments [3] [4], the head movement was assumed to be periodic with a constant distance between the head and the target. It thus makes sense to use a periodic solution for the system. Martin et al. proposed to decompose the joint positions in Fourier series.

$$
\theta_{1}(t)=\frac{a_{01}}{2}+\sum_{k=1}^{N}\left(a_{k 1} \cos (k \omega t)+b_{k 1} \sin (k \omega t)\right)
$$

where $a_{k 1}$ and $b_{k 1}$ are the ankle amplitude coefficients for the $k^{t h}$ harmonic, $\omega$ the pulsation of the target motion and $N$ the number of harmonics in Fourier series.

The system constraints are thus to keep a constant distance between the head and the sinusoidal target (eq.8) and to hold the orthostatic balance (eq.9), i.e, to maintain the CoP inside the base of support (BoS).

$$
\begin{gathered}
A_{t}=l_{1}\left(\cos \left(\theta_{1}\left(\frac{T_{t}}{2}\right)\right)-\cos \left(\theta_{1}(0)\right)\right)+ \\
l_{2}\left(\cos \left(\theta_{1}\left(\frac{T_{t}}{2}\right)+\theta_{2}\left(\frac{T_{t}}{2}\right)\right)-\cos \left(\theta(0)+\theta_{2}(0)\right)\right) \\
C o P_{\min } \leq C o P \leq C o P_{\max }
\end{gathered}
$$

where $\frac{T_{t}}{2}$ is the half period of the target motion, $A_{t}$ its amplitude, $C o P_{\max }$ and $C o P_{\min }$ the upper and the lower BoS limits, respectively.

In the litterature, it is often assumed [6] that the human body tries to minimize the global torque variations. For this reason, (eq.10) is named the minimum torque change criterion.

$$
J_{n}=\sum_{j=1}^{n}\left(\frac{d \Gamma_{1}^{2}}{d t}\left(t_{j}\right)+\frac{d \Gamma_{2}^{2}}{d t}\left(t_{j}\right)\right) \Delta t
$$

where $n$ is the number of discrete time $t_{j}$ and $\Delta t$ the time discretization step, and 10 is the criterion in discrete time. The optimization problem boils down to find the optimal Fourier coefficients that respect these constraints.

\section{Simulation OF THE TRACKING TASK}

\section{A. Simulation parameters}

For the tracking task simulation, we used two different models of humanoid robot, HRP2 and HOAP3. HRP2 $(1.54 \mathrm{~m}$, $58 \mathrm{~kg}$ ) has the mass and the length repartition similar to the Winter's anthropometric model [8]. HOAP3 however is not close to the anthropometric tables $(70 \mathrm{~cm}, 8.8 \mathrm{~kg})$. It is interesting to see how the optimization evolves with a nonstandard anthropometric model.

The initial posture parameters all positioned the double inverted pendulum in an upright position. We took in (eq. 7) only the first Fourier harmonic, to keep a periodic solution. Simulations were performed with different input parameters.
The amplitude of the target varied between 20 and $100 \mathrm{~mm}$, and the oscillation frequencies varied from $0.2 \mathrm{~Hz}$ to $1.2 \mathrm{~Hz}$. The length of the BoS must also be a variable parameter, because in the model the detachment of the sole is not considered. The humanoid feet are rigid, wich can introduce a bouncing phenomenon. It is necessary to adapt the length of the BoS. The difference in the phase between the two joints was measured by using the Lissajous curves.

\section{B. Simulation results}
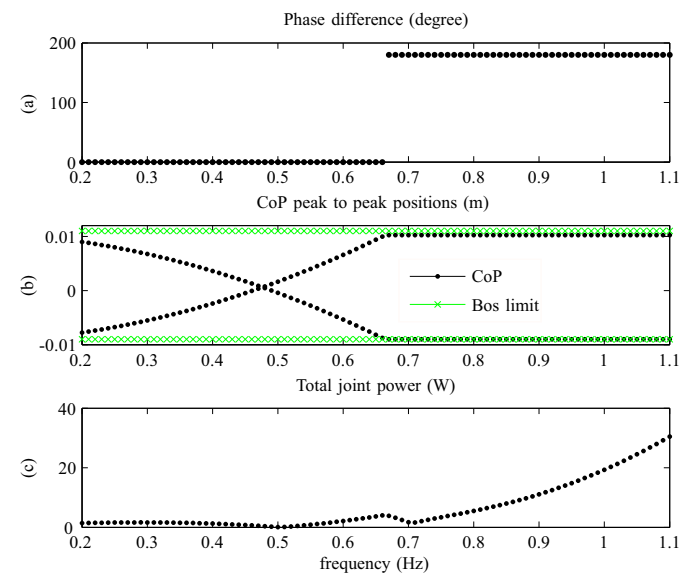

Fig. 3. Typical simulations $\left(a_{T}=4 \mathrm{~cm}, B o S=2 \mathrm{~cm}\right.$ ) for the HRP2 model. In-phase $(f<0.67)$ and anti-phase $(f>0.67)$ coordination modes (a), CoP under the BoS limits for the in-phase mode with a minimum variation at $\omega_{0}$ and on the BoS limits after the phase transition (b), the total net joint power (b) with a inflection close to $\omega_{0}(\mathrm{c})$.

For the model corresponding to HRP2 parameters, two distinctive stable modes appeared (Fig. 3a), similar to the outcome of the anthropometric model used by Martin: the inphase mode between the ankle and the hip at low frequencies and the anti-phase mode at high frequencies. Around the natural frequency $\omega_{01}$, and independently of the length of the $\mathrm{BoS}$ and $A_{t}$, a minimum peak-to-peak variation of the CoP was visible (Fig. 3b) with a reduction of the total joint power used (Fig. 3c).

For the model corresponding to the HOAP3 parameters, two distinctive stable modes again appeared (Fig. 4a), but an antiphase mode was also the preferred mode at very low frequencies, probably due to the non-antropometric parameters. With this exception, all previous observations were confirmed. It is interesting to note that a modification of the inertia and CoM position of each segment, to approach the Winter's parameters [8], produced results closer to the HRP2 simulations.

(Fig. 5), (Fig. 6) illustrate Fourier's first optimal coefficients (i.e the amplitude) for fixed length of BoS and $A_{t}$. These curves, constant before the transition, can be approximated to provide a direct relation between the frequency and the optimal amplitude.

Moreover the size of the constraints $\left(\mathrm{BoS}\right.$ and $\left.A_{T}\right)$ influenced the frequency of phase transition.

There is converging evidence from these results that the CoP governs the emergence of the coordination mode; when CoP 

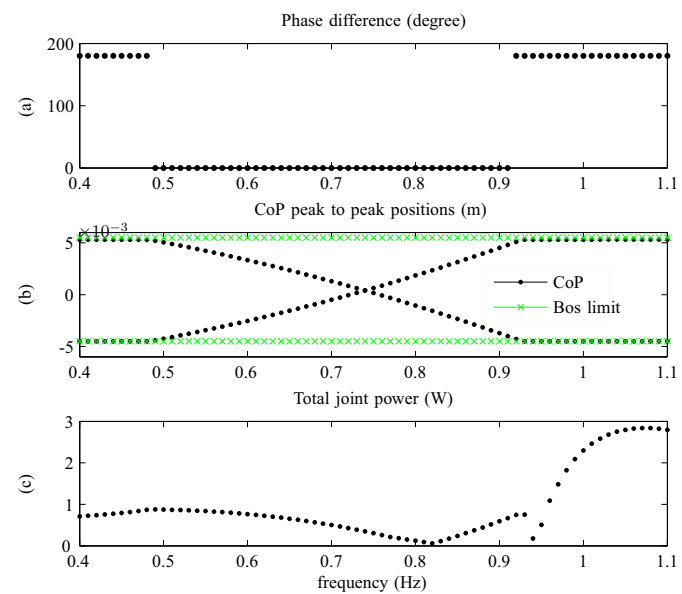

Fig. 4. Typical simulations $\left(a_{T}=4 \mathrm{~cm}, B o S=2 \mathrm{~cm}\right)$ for the HOAP3 model. Two coordination modes anti-phase $(f<0.47, f>0.92)$ and inphase $(0.47<f<0.92)$ (a), $\mathrm{CoP}$ with a minimum variation close to $\omega_{0}$ (b), the total net joint power (b) with a inflection close to $\omega_{0}$ (c), the two last curves (d) and (e) represent the optimal amplitude (first Fourier coefficients.) at each frequency

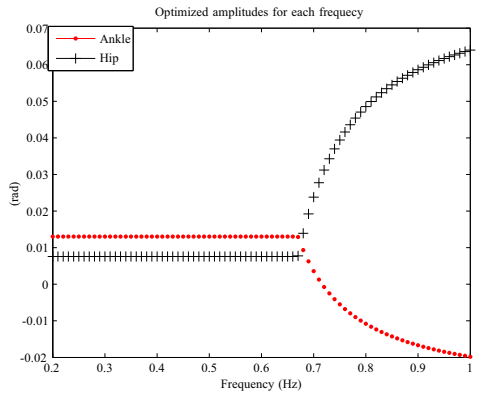

Fig. 5. Optimized amplitudes for the HRP2 model. Amplitudes were constant before the phase transition but increased afterwards with the antiphase mode.

approaches the $\mathrm{BoS}$ limit, the hip-ankle relative phase switches to a new value. The anti-phase mode thus appears to be the most stable mode in terms of balance, whereas the in phase mode appears to be the mode minimizing energy.

These results also tend to show that the natural frequency is a privileged frequency at which the system needs minimum joint power and has a better equilibrium(eq. 3). The second natural frequency has no physical sense since the constraints are activated and change the model used for calculation of the natural frequencies. The second minimum on the joint power curves, located just after the transition, is due to the ankle torque. In fact, the torque produced by the in phase mode near the transition is important (in order to maintain balance), which is not the case after the transition.

In conclusion, we observed a decrease in the peak-to-peak CoP movement during the anti-phase mode, a reduction in the total joint power for the in phase mode, and an increase in balance with a minimization of energy around the natural frequencies.

\section{EXPERIMENTAL RESUlTS AT THE TRACKING TASK}

To show the effectiveness of the proposed optimized biomechanical model, we implemented in HOAP3 and HRP2 the

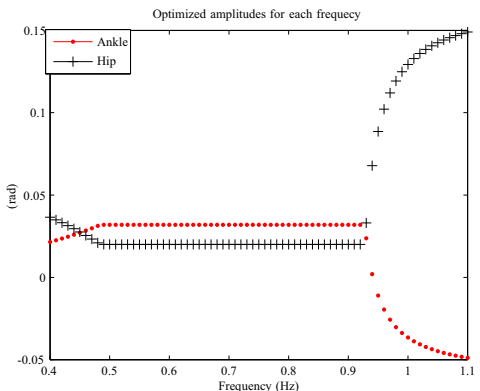

Fig. 6. Optimized amplitudes for the HOAP3 model. The anti-phase mode was observed at very low frequencies $(f<0.47 \mathrm{~Hz})$ due to the specific HOAP3 parameters; then amplitudes followed the HRP2 amplitude pattern.

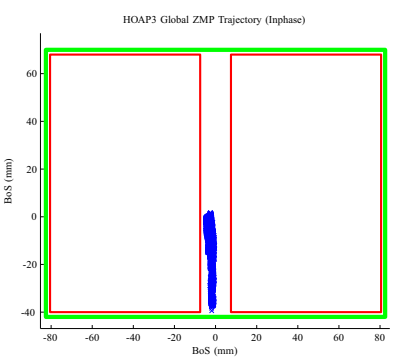

(a)

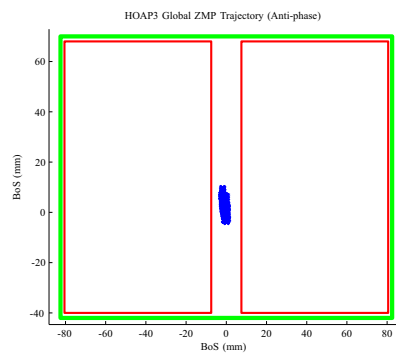

(b)
Fig. 7. CoP evolution for HOAP3 (BoS top view) showing a better stability for the anti-phase mode(b) than for the in-phase mode (a).

offline optimal joint amplitudes calculated for each frequency of the target motion. The robots were used without stabilizers and positioned initially in an upright position. The main features we expected to observe were the minimization of the joint energy during the in phase mode, the minimization of the CoP peak-to-peak displacement during the anti-phase mode, the shaping role of the CoP location in the phase transition, and the $\mathrm{CoP}$ and torque values around the natural frequencies.

\section{A. Effects of the coordination mode on the CoP}

To analyse the influence of the two coordination modes on the CoP position, we executed the same head motion at various frequencies. The sign of the hip coefficient was changed and consequently the coordination bifurcated from in phase to antiphase. On (Fig. 7) we can see clearly that the anti-phase mode reduced the CoP peak-to-peak displacement of the HOAP3 robot.

CoP evolution of HRP2 (Fig. 8) described the same phenomenon.

The anti-phase mode thus appears to improved the general equilibrium in both robots.

\section{B. Effects of the coordination mode on the energy}

In this part, only the HRP2 results are presented since the robot HOAP3 has no joint torque sensors. The methodology was identical to the one used for the $\mathrm{CoP}$ analysis.

The in-phase mode (Fig. 9a,b) dissipated less joint power than the anti-phase mode (Fig. 9c,d). This was due to the hip 

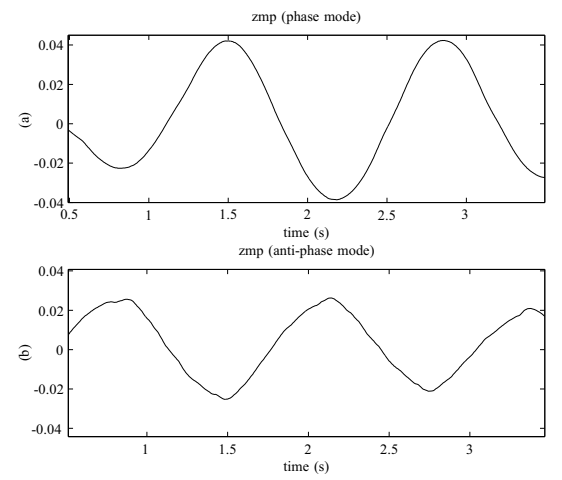

Fig. 8. CoP evolution during two periods of head oscillation for HRP2 $\left(A_{T}=2 \mathrm{~cm}, B o S=2 \mathrm{~cm}\right)$; the anti-phase mode reduces the CoP peak-topeak distance.
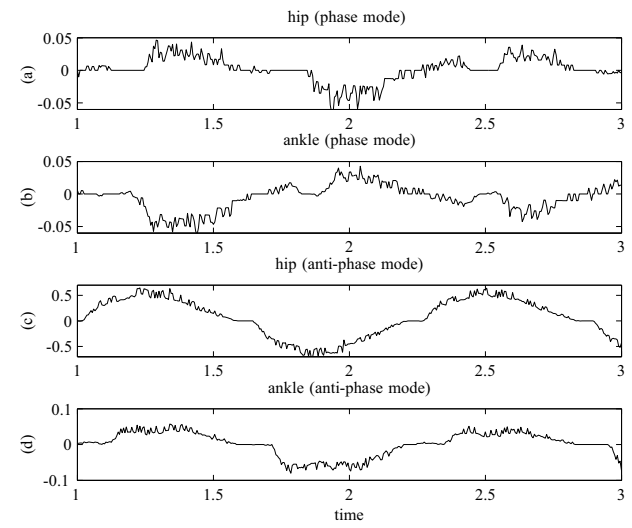

Fig. 9. The joint power (W) is inferior for the in-phase mode (c,d) compared to the anti-phase mode $(\mathrm{a}, \mathrm{b})$. Ankle power is slightly the same for in phase mode (a) and anti-phase mode (d), hip power during anti-phase becomes larger in the movement.

joint which increased after the phase transition. This shows clairly that the in-phase mode minimizes joint power.

\section{The role of CoP position in phase transition}

In section III, we evidenced that the CoP dynamics governs both the general equilibrium and the coordination mode adopted. We tried to evidence this effect with humanoid robots by observing when the CoP location crossed the BoS limits. Because however we did not take into account a possible takeoff of the sole from the ground, rebound behavior sometime appeared due to the rigidity of the humanoid feet. Thus, robots could fall because of these uncontrolled rebounds. We thus searched for a compromise between the length of the BoS and the amplitude of target motion. The experiment was performed with short lengths of BoS. For example, with HOAP3, a BoS length of $1 \mathrm{~cm}$ together with a head amplitude of $5 \mathrm{~cm}$, produced a stable value of the in phase mode at $0.85 \mathrm{~Hz}$ while the predicted value was $0.92 \mathrm{~Hz}$. As an other example using HRP2 $\left(B o S=2 \mathrm{~cm}\right.$ and $\left.A_{T}=4 \mathrm{~cm}\right)$, the experimental in phase stable value was around $0.55 \mathrm{~Hz}$ while the predicted value was $0.69 \mathrm{~Hz}$. The difference between experimental and predicted results was more important for HRP2 than for
HOAP3, probably because HRP2 has a compliance element located at is ankle level. This compliant element can add oscillations which are not considered in our model. The differences can also be due to an incomplete model: the missing friction parameter, among others, may reduce these differences. Above these values the only stable mode was the anti-phase mode. This confirms that the $\mathrm{CoP}$ is an important variable constraining the adopted coordination pattern.

\section{Robot postural states around natural frequencies}

Section III evidenced privileged frequencies, with minimal variations in peak-to-peak $\mathrm{CoP}$ and energy.

HRP2 sensors provide the joint torques as function of frequency. Our experiments did not reveal a direct effect of natural frequencies, but a slow down in the increasing of torques with frequency near the theoretical natural frequency. Further tests are necessary in order to fully understand the effects of natural frequencies.

For the CoP, the test is performed with HOAP3.

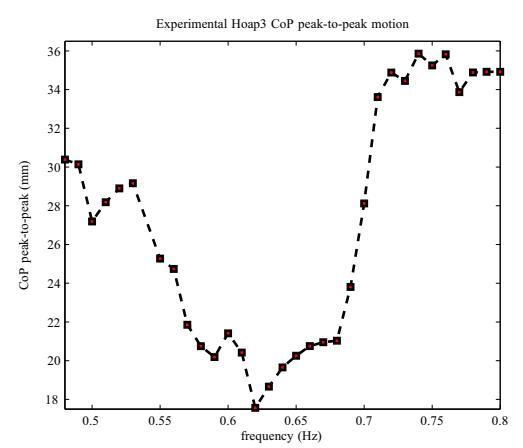

Fig. 10. Evolution of the experimental CoP peak-to-peak displacement as a function of frequency. A minimum variation observable at $0.62 \mathrm{~Hz}$ (not depending of the $\mathrm{BoS}$ and the $A_{T}$ ) but less important than the theoretical frequency.

A diminution of the peak-to-peak $\mathrm{CoP}$ displacements is visible (Fig. 10), but less important and not at the theoretical frequency. That is certainly due to the imprecisions of the model (section .IV-C). Nevertheless, the important fact is the presence of a privileged frequency.

\section{DisCUSSION AND PERSPECTIVES}

By using humanoid robots we validated the results obtained by Bardy et al. [4]. We showed experimentally, that the antiphase postural mode is more efficient for the global stability of the postural system at high frequencies, and that the in phase mode minimizes joint power at low frequencies.

Phase transition occurred when CoP reached the limits of the $\mathrm{BoS}$. This proves that the distance between the $\mathrm{CoP}$ and the limits of the BoS is a key variable which has to be taken into account by the nervous system.

The natural frequency for in-phase mode appears like a privileged mode in terms of CoP displacements and in terms of joint power. Based on these first results, we aim at developing a ankle-hip controller which takes into account the natural frequencies. For example, we would like to generate adequate 
trajectories in order to maintain the frequency spectrum close to the natural frequency.

However, the model analysed in this paper does not take into account all the dynamical phenomena observed in humans.

For this reason, and also in order to improve the humanoid robot control, we plan to develop a more complete model of the human postural system, capitalizing on nonlinear coupled oscillators (ankles and hips). Haken Kelso and Bunz (HKB) have proposed a nonlinear coupled-oscillators system for bimanual coordinations [10]. The HKB model is able to produce bi-stability, phase transition, amplitude evolution with frequency increase. With few changes, such as those proposed by [11], we plan to have a more complete picture of human postural coordination, in order to create a new controller for the ankles and the hips.

The use of nonlinear coupled oscillators associated with the results obtained by the biomechanical model will be proposed to create a new controller between the ankle and the hip.

This controller will be able to automatically change the phase difference, the joint amplitude necessary to maintain balance, capitalizing on the natural frequency properties.

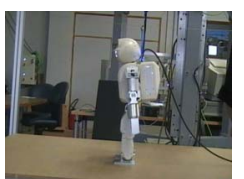

(a) $\mathrm{t}=0 \mathrm{sec}$

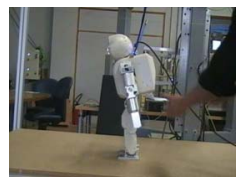

(d) $\mathrm{t}=0,75 \mathrm{sec}$

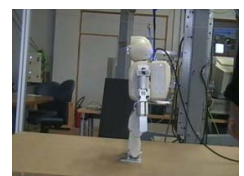

(b) $\mathrm{t}=0,25 \mathrm{sec}$

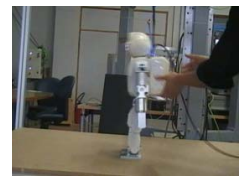

(e) $\mathrm{t}=1 \mathrm{sec}$

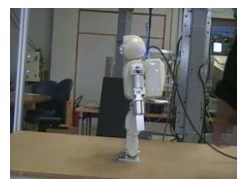

(c) $\mathrm{t}=0,5 \mathrm{sec}$

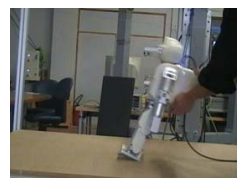

(f) $\mathrm{t}=1.45 \mathrm{sec}$
Fig. 11. HOAP3 experiments with in-phase coefficients at high frequency $\left(f=1 \mathrm{~Hz}, B o S=1 \mathrm{~cm}, A_{t}=5 \mathrm{~cm}\right)$. The robot cannot maintain its balance and falls backward.

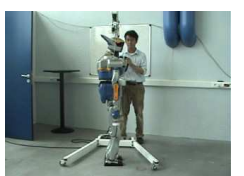

(a) $\mathrm{t}=0 \mathrm{sec}$

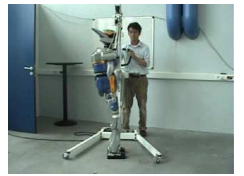

(d) $\mathrm{t}=1.1 \mathrm{sec}$

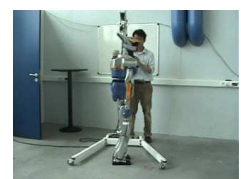

(b) $\mathrm{t}=0.4 \mathrm{sec}$

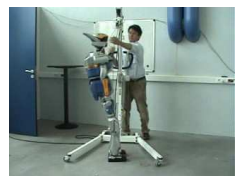

(e) $\mathrm{t}=1.15 \mathrm{sec}$

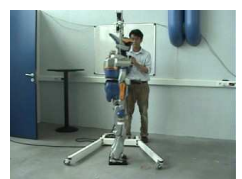

(c) $\mathrm{t}=0.8 \mathrm{sec}$

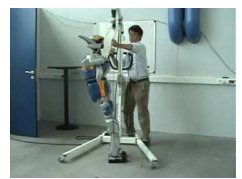

(f) $\mathrm{t}=1.2 \mathrm{sec}$
Fig. 12. HRP2 experiments with in-phase coefficients at high frequency ( $\left.f=0.6 \mathrm{~Hz}, B o S=2 \mathrm{~cm}, A_{t}=4 \mathrm{~cm}\right)$. The robot cannot maintain its balance and falls backward.

\section{ACKNOWLEDGMENT}

Author Nacim Ramdani is on leave from CERTES EA 3481 Universite Paris 12 Val de Marne.

\section{REFERENCES}

[1] L.M. Nashner, G. McCollum, The organization of postural movements: a formal basis and experimental synthesis, Behavioral and Brain Sciences, vol. 26, pp. 135-172, 1985.

[2] JA.S. Kelso, Dynamics Patterns, The Self-Organization of brain and behavior, The MIT Press, 1995.

[3] B. G. Bardy, L. Marin, T. A. Stoffregen, R. J. Bootsma, Postural coordination modes considered as emergent phenomena, J Exp Psychol Hum Percept Perform., vol. 25, pp. 1284-1301, 1999.

[4] B.G Bardy, O. Ouillier, R. J. Bootsma, T.A. Stoffregen, Dynamics of Human Postural Transitions, J Exp Psychol Hum Percept Perform., vol. 28, No. 3, pp. 499-514, 2002.

[5] L. Martin, V. Cahouet, M. Ferry, F. Fouque, Optimization model predictions for postural coordination modes, Journal of Biomechanics, vol. 39,pp. 170-176, 2006.

[6] E.S. Engelbrecht1, J.P. Fernandez, Invariant characteristics of horizontalplane minimum-torque-change movements with one mechanical degree of freedom, Biological Cybernetics, vol. 76, pp. 321-329, 2004.

[7] V. Cahouet, L. Martin, D. Amarantini, Static optimal estimation of joint accelerations for inverse dynamics problem solution, Journal of Biomechanics, vol. 35, pp. 1507-1513, 2002.

[8] D.A. Winter, Biomechanics and motor control of human movement third edition, Ed. Wiley, 2005.

[9] M. Williamson, Robot Arm Control Exploiting Natural Dynamics, Ph.D. Thesis, MIT Department of Electrical Engineering and Computer Science, 1999.

[10] H. Haken, J.A.S. Kelso, H. Bunz, A theorical model of phase transitions in human hand movements,Biological Cybernetics, vol. 51, pp. 347-356, 1985.

[11] A. Fuchs , V. Jirsa, The HKB model revisited: How varying the degree of symmetry controls dynamics, Human Mouvement science vol. 19, pp. 425-449, 2000. 